\section{Implementing clinical guidelines}

\section{Has a lot to offer patient care}

EDrroR,-We are disappointed at the negative tone of Tony Delamothe's editorial on clinical guidelines and feel that it does not address some important issues. ${ }^{1}$ The Scottish Office definition he quotes is too narrow. Much improvement in health care would be achieved if we made explicit and standardised our treatment programmes. As health care is one of the more complex multidisciplinary processes, ${ }^{2}$ such "guidelines" must cover the activity of the entire health care team. The process of drawing such guidelines has many advantages. It clarifies the areas where there is genuine discretion and the need for decision making. If the entire clinical team is involved a high level of commitment is obtained. Patient care is standardised and a yardstick for the measurement of performance is provided. It becomes possible to identify the areas where there are clear issues about "best practice." At Central Middlesex Hospital NHS Trust during 1989-91 several medical guidelines were written, and their use was evaluated with both qualitative and quantitative methods. ${ }^{34}$ We too found that their impact was limited.

Central Middlesex Hospital is committed to refocusing its services around the patient (the adoption of patient focused care). One of the key vehicles for change has been the development and implementation of "multidisciplinary care protocols." These have been written and agreed by all members of the team and incorporate national or local medical guidelines. They have been designed to become part of the actual unitary record of care. They are not cast in stone. All professional staff retain the right to seek change or update the agreed plan. Variations from the care protocol are noted during the routine practice of care and then are aggregated and used for clinical audit purposes. We use this information to validate both our guidelines and our care. The protocol is shared with patients, and we have found it to increase rather than diminish their confidence. The process does not detract from clinical freedom; rather, it identifies the critical points where decisions are required, surely the essence of the medical input. There are many areas where patient care would benefit greatly from establishing guidelines. It would be unfortunate if a too scrupulously scientific approach held back a process which has so much to offer to patient care.

MARTIN MCNICOL AMANDA LAYTON GRAHAM MORGAN

Central Middlesex Hospital NHS Trust, London NW10 7NS

1 Delamothe $\mathrm{T}$. Wanted: guidelines that doctors will follow. $B M Y$ 1993;307:218. (24 July.)

Arvec to anthors
Priority will be given to letters that are less
than 400 words long and are typed with
double spacing. All authors should sign the
letter. Please enclose a stamped addressed
envelope for acknowledgment.

$2 \mathrm{McNicol} \mathrm{M}$. Achieving quality improvements by structured patient management. $Q H C$ 1992;1(suppl):40-1.

3 Gabbay J, Layton AJ. Evaluation of audit of medical records in district general hospital. QHC 1992;1:43-7.

4 Bell D, Layton AJ, Gabbay J. Use of a guideline based questionnaire to audit hospital care of acute asthma. BMJ 1991;302:1440-3.

\section{It works in Australia}

EDrToR,-Tony Delamothe asks for guidelines that doctors will follow. ${ }^{1}$ Prescribing guidelines have been successful in Australia.

The enterprise began in Melbourne in 1978 with the publication of Antibiotic Guidelines, written by an independent group of microbiologists chaired by a clinical pharmacologist. With the imprimatur of the state health authority these guidelines gained sufficient acceptance to require many subsequen editions. They have been joined by Analgesic Guidelines, Psychotropic Drug Guidelines, and Cardiovascular Drug Guidelines. Guidelines on gastrointestinal and respiratory drugs will be available next year.

The format of the booklets parallels clinical practice. It proceeds from diagnosis to treatment, giving succinct, explicit recommendations for treating identifiable entities with brief discussion to orient the prescriber. The guidelines target community as well as hospital prescribers and are marketed vigorously to the profession at comparatively low price (\$A15). With about 35000 books being sold annually, the enterprise is almost self supporting. Large sales imply acceptance but do not ensure use. Studies have found, however that concordance with recommendations for treatment has been as high as $76 \%$ in a teaching hospital $^{2}$ and $60 \%$ in general practice. ${ }^{3}$

The format chosen demands that recommendations should be made regardless of whether there is documented evidence for any particular treatment This reflects the prescriber's problem. Members of the group that writes the guidelines are chosen to combine scholarship with practical experience plus a willingness to challenge conventional wisdom Literature searches in all controversial or rapidly developing topics underpin decisions of the groups. Where the evidence is not clear, recommendation are based on consensus. Criteria here include the profile of side effects, data on long term safety, and the cost. All recommendations are subject to biennial review, and feedback from users is actively sought so that the publications can be refined.

The guidelines thus give prescribers a second opinion which is based on rigorous, but not doctrinaire, assessment of current knowledge and practice. They have penetrated deep into Australian medical practice and are increasingly taken into account by regulatory and reimbursement authorities and the pharmaceutical industry.

MAY HEMMING

Victorian Drug Usage Advisory Committee, Carlton South 3053,

$$
\text { Australia }
$$

ML MASHFORD

Victorian Medical Postgraduate Foundation,

Therapeutics Committee,

North Melbourne 3051,

Australia

1 Delamothe T. Wanted: guidelines that doctors will follow. $B M$ 1993;307:218. (24 July.)

2 Raymond PM, Robertson MB, Mashford ML. A decade of antibiotic use. Med f Aust 1989;150:619-26.
3 Landgren FT, Harvey KJ, Mashford ML, Moulds RFW, Guthrie B, Hemming $M$. Changing antibiotic prescribing by educational marketing. Med f Aust 1988;149:595-9.

\section{Guidelines may not be cost effective}

EDITOR,-Tony Delamothe mentioned the relevance of guidelines to clinical outcome on two brief occasions. ${ }^{1}$ Indeed, clinical guidelines will be cost effective only if the resources spent to develop and disseminate them are justified by reductions in health care expenditure or improvements in patients' outcomes. There is very little evidence so far that guidelines are helpful in terms of cost containment. Guidelines organised around particular diagnostic and therapeutic procedures could possibly reduce expenditure in a limited number of areas by presenting appropriate and inappropriate indications for their use. ${ }^{2}$ Guidelines dealing with the diagnosis of people with particular symptoms and management of people with known conditions can be considered valid only if the desired patient outcome is achieved, and often outcome data that closely correlate with the guideline's process of care are lacking. Unless guidelines become more focused on clinical outcome and on site clinical evaluations have tested their validity, efficacy, and applicability, health professionals will continue to show some reluctance to follow them.

Despite these problems there are guidelines for effective interventions that have been well researched, such as those on effective care in pregnancy and childbirth. ${ }^{3}$ Guidelines like these are clearly not "intellectually suspect" and they do not "formalise unsound practice." The local acute provider units in south east London have been very cooperative in detailing the guidelines and outcome indicators that they are using and intend to use. Clinical guidelines may be more useful for junior medical staff, who may not perceive clinical freedom in quite the same way that the editorial implied their seniors do. If there were any areas of clinical practice where nationally recommended effective interventions were not being used, purchasers would have a clear duty, in the interests of the population that they serve, to ensure that guidelines for such interventions were adopted by clinicians.

Clinical guidelines also have implications for consumers and for future research. In 1989 the Agency for Health Care Policy and Research was established in the United States to enhance the quality, appropriateness, and effectiveness of health care services and access to these services. The agency commissioned multiagency expert panels to develop clinical practice guidelines which have been produced on several areas. The purpose of the guideline, for instance, on urinary incontinence was "to improve diagnosis and treatment of urinary incontinence; reduce variations and thereby improve the quality of patient care in clinical practice; educate health professionals and consumers about the condition; and encourage further biomedical, clinical, and cost research on the topic."

Maybe the title of Delamothe's editorial should have been "Wanted: guidelines that health professionals and consumers will follow."

ALASTAIR MCCOLI 